The Astrophysical Journal, 308:L11-L15, 1986 September 1

(C) 1986. The American Astronomical Society. All rights reserved. Printed in U.S.A.

\title{
PULSED TeV GAMMA RAYS DETECTED FROM HERCULES X-1 DURING X-RAY SOURCE ECLIPSE
}

\author{
P. W. Gorham, ${ }^{1}$ M. F. Cawley, ${ }^{2}$ D. J. Fegan, ${ }^{3}$ K. G. Gibbs, ${ }^{4}$ R. C. Lamb, ${ }^{2}$ \\ D. F. Liebing, ${ }^{2,5}$ N. A. Porter, ${ }^{3}$ V. J. Stenger, ${ }^{1}$ and T. C. Weekes ${ }^{4}$ \\ Received 1986 April 3; accepted 1986 May 29
}

\begin{abstract}
We have detected distinct $0.808 \mathrm{~Hz}$ pulsations from Her X-1 in 1985 June using the atmospheric Cherenkov technique with the Whipple Observatory $10 \mathrm{~m} \gamma$-ray telescope, which had an effective $\gamma$-ray energy threshold of $\sim 0.6 \times 10^{12} \mathrm{eV}$. The $2 \mathrm{hr}$ observation spanned the transition into X-ray eclipse, at which the $\mathrm{X}$-ray source Her $\mathrm{X}-1$ is first occulted by the companion star HZ Herculis at orbital phase $\sim 0.93$ in the binary system. The pulsation persists for $\geq 1 \mathrm{hr}$ after the beginning of the $\sim 6 \mathrm{hr}$ eclipse, and we conclude that the $\gamma$-ray production site is in this case not coincident with the $\mathrm{X}$-ray source.
\end{abstract}

Subject headings: gamma-rays: general — pulsars — stars: individual $-\mathrm{X}$-rays: binaries

\section{INTRODUCTION}

In a forthcoming paper (Gorham et al. 1986, hereafter Paper I), we present evidence for repeated episodes of emission of $\sim 10^{12} \mathrm{eV}(1 \mathrm{TeV}) \gamma$-rays from the $1.24 \mathrm{~s} \mathrm{X}$-ray pulsar Hercules $\mathrm{X}-1$, which confirms an observation by Dowthwaite et al. (1984) of a 3 minute outburst of $\mathrm{TeV}$ $\gamma$-rays from this source in 1983. These observations have all used the atmospheric Cherenkov technique (ACT) to detect extensive air showers produced by the primary source photons and have relied on Fourier analysis of the resulting time series to distinguish the coherent pulsations from the random cosmic-ray background. The power of these techniques to detect a periodic signal in the case of small signal-to-noise ratios has been amply demonstrated in other non-X-ray detections of pulsations from this source; for example, in optical observations (Davidsen et al. 1972; Groth 1974; Middleditch and Nelson 1976), and also in recent optical/ infrared observations (Middleditch, Pennypacker, and Burns 1983; Middleditch, Puetter, and Pennypacker 1985).

In this Letter we report on one of a number of further episodes of emission from Her X-1 at energies in the TeV range. We have selected this episode specifically because it occurs during the nominal X-ray eclipse of Her X-1, which takes place every $\sim 1.7$ days when the $\mathrm{X}$-ray intensity drops to a value which indicates that the primary $X$-ray source is completely obscured by the companion star. In this 1985 June $16 \gamma$-ray emission episode, our observation spans an eclipse transition, and we find that significant $\gamma$-ray fluxes appear only after the eclipse has begun and persist for at least $\sim 1$ $\mathrm{hr}$ thereafter. We conclude that the $\mathrm{X}$-ray and $\gamma$-ray production sites are, in this instance at least, not geometrically coincident in this binary system. We also discuss implications

\footnotetext{
${ }^{1}$ High Energy Physics Group, University of Hawaii.

${ }^{2}$ Department of Physics, Iowa State University.

${ }^{3}$ Department of Physics, University College Dublin.

${ }_{5}^{4}$ Harvard-Smithsonian Center for Astrophysics.

${ }^{5}$ Presently at University of Utah, Salt Lake City.
}

for models (cf. Eichler and Vestrand 1985) which postulate a high-energy particle beam as the source of the $\geq \mathrm{TeV} \gamma$-rays.

\section{OBSERVATIONS}

We tracked Her X-1 continuously for $2 \mathrm{hr}$ on the night of 1985 June 16, and we digitized arrival times of the $\sim 10 \mathrm{~ns}$ Cherenkov pulses from all extensive air showers originating from primary cosmic rays of energy $\gtrsim 0.6 \mathrm{TeV}$ (Hillas 1985), within $\sim 1$. . 5 of the source direction, to $\sim 1 \mu \mathrm{s}$ precision. The resulting time series conforms statistically to that expected from a Poisson process with a mean rate of typically $\sim 3 \mathrm{~Hz}$. Applications of Fourier techniques to such series have been discussed by Leahy et al. (1984) and Gorham (1986); see also Middleditch (1976).

Due to observing convention, a data gap of $\sim 1$ minute duration occurred every 30 minutes; however, the accuracy of the relative timing was preserved throughout the observation. In our usual ACT operation, the telescope is shifted off-source every 28 minutes to more accurately determine the background rate. This was not done here in order to ensure more continuous sensitivity in time, since Her X-1 has exhibited short time scale activity in $\gamma$-rays in the past (Paper I; also Dowthwaite et al. 1984). Thus the background uncertainty limits the accuracy of our flux estimates. However, the timing analysis of the more continuous data is simplified, since the data gaps, and their undesirable effects on the power spectrum, are minimized. For further details of the data acquisition and reduction, we refer the reader to Paper I.

Although Doppler-shift frequency variations are not large for time series of these durations, we corrected the raw arrival times to the solar system barycenter, and then to the Her X-1/HZ Her barycenter, to remove effects of the orbital motions of Earth and Her X-1. We used the formula and ephemeris of Deeter, Boynton, and Pravdo (1981) for these corrections. The radial component of the pulsar velocity varies from -90 to $-40 \mathrm{~km} \mathrm{~s}^{-1}$ during our observation; this 
velocity change corresponds to a frequency shift of $0.17 \mathrm{mHz}$ which exceeds the frequency resolution intrinsic to the $\sim 7000 \mathrm{~s}$ duration of our observation (thus we take the time corrections as necessary to preserve the pulse phase). We also padded the $\sim 1$ minute data gaps with a constant value to eliminate any spurious sidelobes they might cause in the spectrum (cf. Paper I). After these corrections we Fourier- transformed the time series and calculated the power spectrum in the vicinity of the $0.808 \mathrm{~Hz}$ first harmonic of the pulsar frequency.

\section{RESULTS}

Table 1 summarizes some of the important features of this observation. Figure $1 a$ shows the minute-by-minute count rate

TABLE 1

1985 June 16: Hercules X-1 Gamma-Ray Observation Details

\begin{tabular}{|c|c|c|c|c|c|c|}
\hline \multirow{3}{*}{$\begin{array}{c}\text { JULIAN } \\
\text { DATE } \\
(-2,446,065.5)\end{array}$} & \multirow{3}{*}{$\begin{array}{l}\text { EMISSION } \\
\text { DURATION } \\
\text { (s) }\end{array}$} & \multirow{2}{*}{\multicolumn{2}{|c|}{ Phases }} & \multicolumn{2}{|c|}{ FREQUENCIES } & \multirow{3}{*}{$\begin{array}{c}\text { FLUX } \\
\left(\mathrm{cm}^{-2} \mathrm{~s}^{-1}\right)\end{array}$} \\
\hline & & & & Expected $^{\mathrm{c}}$ & Measured $^{\mathrm{d}}$ & \\
\hline & & Orbital $^{\mathrm{a}}$ & 35 Day $^{b}$ & $(\mathrm{~Hz})$ & $(\mathrm{Hz})$ & \\
\hline 167.20851 . & $\sim 4300$ & $0.914-0.962$ & 12.73 & $0.807896(3)$ & $0.808586(27)$ & $1.5 \times 10^{-10}$ \\
\hline
\end{tabular}

${ }^{\mathrm{a}}$ Eclipse phases span the interval $0.9317-0.0683$.

bUsing a turn-on time (defined as phase 0.0 here) of JD 2,445,788.0 (Ögleman et al. 1985) and period of 34.928. Integer part indicates number of cycles from $T_{0}$.

${ }^{c}$ Extrapolated from X-ray results of Ögelman et al. 1985, with standard error in $\mu \mathrm{Hz}$ (due to uncertainty in pulsar period derivative) in parentheses.

${ }^{\mathrm{d}}$ Standard error in $\mu \mathrm{Hz}$ in parentheses.

eApproximately 400 photons detected in an interval of $\sim 4300 \mathrm{~s}$ after eclipse, with a collection area of $\sim 6 \times 10^{8} \mathrm{~cm}^{2}$; Hillas 1985 .


FIG. 1.-(a) Time series for the observation of 1985 June 16. Typical standard error is indicated by bar to the left. The gaps at $\sim 30$ minute intervals are artifacts of the data acquisition. The $\sim 100 \mathrm{~s}$ time scale of the eclipse transition is indicated by the dashed vertical lines, and the solid line fit to the pre-eclipse data is that expected from zenith angle effects (described in text); an extrapolation to the data during eclipse is indicated by the dashed extension. (b) Fourier power spectrum for the data preceding eclipse, with the expected X-ray frequency of Her X-1 marked by the arrow, and the horizontal bar above the arrow indicating a $\pm 0.67 \mathrm{mHz}$ band around this frequency. (c) Similar power spectrum for the data during eclipse, showing strong evidence for pulsations within $\sim 0.6 \mathrm{mHz}$ of the expected frequency. 
as a function of time, with the estimated time of the $\sim 100 \mathrm{~s}$ eclipse transition marked by dashed lines, based again on the orbital ephemeris of Deeter, Boynton, and Pravdo (1981). The data gaps every 30 minutes are evident, and the trend toward increasing rate is consistent with zenith angle effects as the source ascends in elevation. The solid portion of the superimposed curve is a fit which is proportional to $\sim(\cos z)^{1.4}$, where $z$ is the zenith angle. We determined this dependence empirically for the night in question using background data obtained from other observations. The dashed portion of the curve is the extension of the pre-eclipse fitted rate to the data during eclipse. We use the pre-eclipse data to establish the background rate for Her X-1 because there appears to be little or no pulsed content in this data, as we show in the following.

In Figures $1 b$ and $1 c$ we plot the power spectra around the expected pulsar frequency (as marked by the arrow) for the data before and during eclipse, respectively. The horizontal bar at the top of the arrow indicates a band of width \pm 0.67 $\mathrm{mHz}$, or $\pm 200 \mathrm{~km} \mathrm{~s}^{-1}$ in terms of source Doppler shifts due to motion relative to Her X-1. This frequency band spans the possible frequency shifts due to the known relative velocities within the Her $\mathrm{X}-1 / \mathrm{HZ}$ Her system and also accounts for possible reconstruction variations in the Fourier transform (cf. Leahy, et al. 1984).
From Figure $1 b$ we find that the maximum power value within $\pm 0.67 \mathrm{mHz}$ of the $\mathrm{X}$-ray frequency in the power spectrum for the data before eclipse is $\sim 3.3$ times the mean noise power level; such a value occurs $\sim 15 \%$ of the time for a random time series in the four trial frequencies in this band. Thus we detect no significant pulsations in the data before eclipse, with a 90\% confidence level upper limit of $7.2 \times 10^{-11}$ $\mathrm{cm}^{-2} \mathrm{~s}^{-1}$ for a pulsed flux. This limit is based on the ratio of power values between the pre-eclipse and post-eclipse data assuming a sine-wave signal.

The peak in the power spectrum in Figure $1 c$ appears at $\sim 0.6 \mathrm{mHz}$ above the expected X-ray frequency of $0.8079 \mathrm{~Hz}$. Such frequency shifts are also suggested in other reported data (see Paper I) and could have physical origins, given the large relative velocities possible in this binary system. The sign of the apparent frequency shift corresponds to material approaching us. The companion star velocity does have a component in this direction during the observation; however, the magnitude of the implied velocity $\left(\sim 200 \mathrm{~km} \mathrm{~s}^{-1}\right)$ appears to be too large to be easily attributable to $\mathrm{HZ} \mathrm{Her.}$

In Figure $2 a$, we plot the same spectrum as Figure $1 c$, now expanded to show approximately 250 independent frequencies around the expected X-ray frequency. Figure $2 b$ is a histogram of all of the power values in Figure $2 a$, which displays
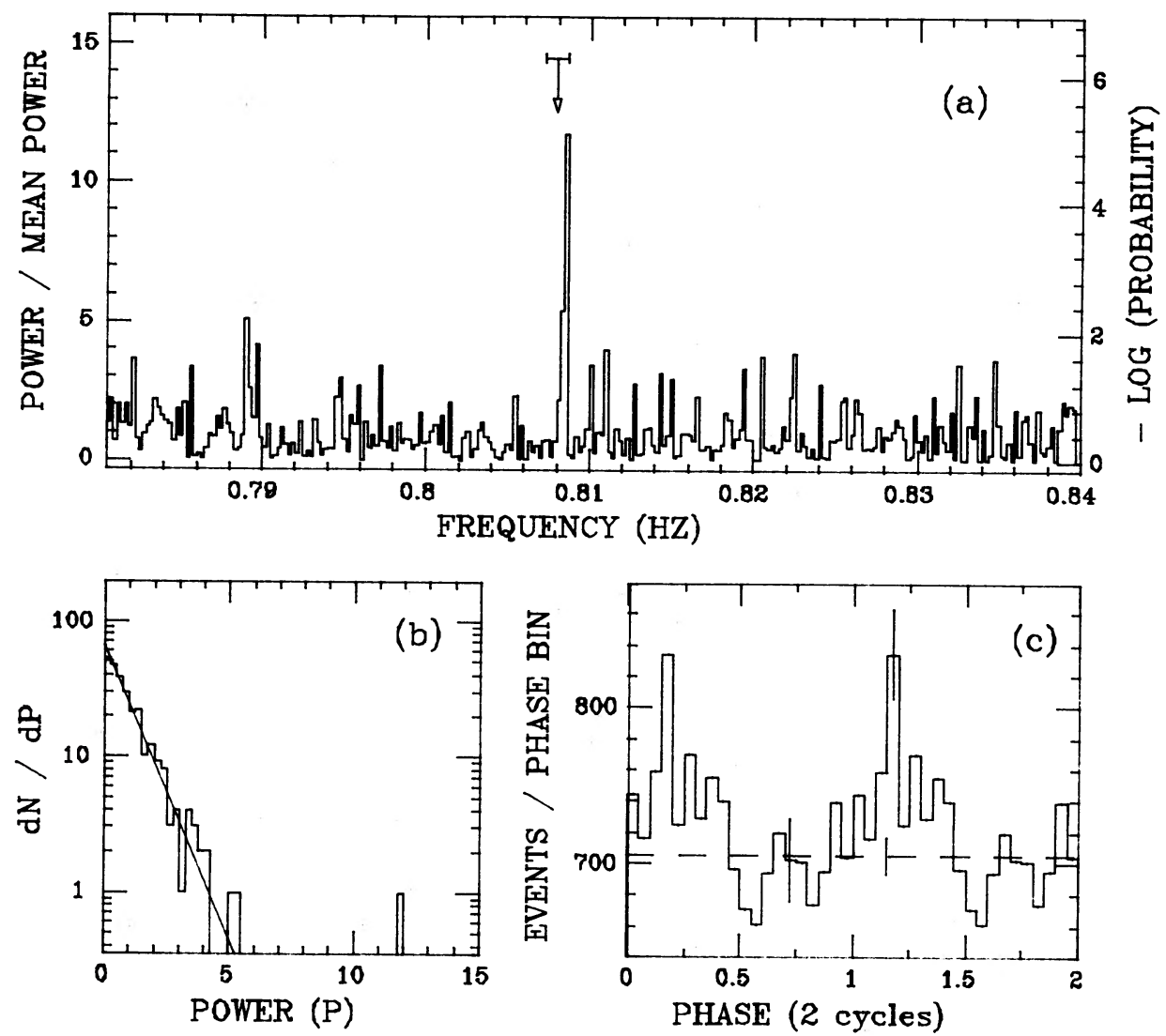

Fig. 2.- (a) Power spectrum of Fig. 1c, expanded to show $\sim 250$ frequency bins around the $0.808 \mathrm{~Hz} \mathrm{X}$-ray frequency, marked by arrow. (b) Distribution of power values in Fig. $2 a$, with a fitted exponential, indicating the expected statistical behavior of the power. Peak at $\sim 0.808 \mathrm{~Hz}$ is indicated by the value near 12. (c) Light curve for the data during eclipse, with estimated background shown with a dashed line. Bin errors are statistical only; background error includes estimated systematic as well as statistical errors. 
the behavior of the power as a test statistic. The fitted exponential has a slope of $-0.99 \pm 0.07$ and a $\chi^{2}$ of 12.3 for 20 degrees of freedom and clearly satisfies the statistical expectations for the spectral power values (cf. Scargle 1982). The value at $\sim 11.9$ has a chance probability of $\exp (-11.9)$ $\approx 7 \times 10^{-6}$ of being observed at a single preselected frequency, and this is increased to $4 \times 10^{-5}$ that it would occur at one of the approximately six independent Fourier frequencies within the $\pm 0.67 \mathrm{mHz}$ band, in data that were entirely random. Figure $2 c$ shows the light curve for these data, folded at the period indicated by the peak in Figure $2 a$.

We have investigated the time dependence of the pulsed flux during the $\sim 70$ minute eclipse observation by dividing the time series into seven $\sim 18$ minute subsegments (which overlap 50\% with each adjacent subsegment in time), and calculating the power ratio at the first four harmonics of the detected frequency as determined by the peak in Figure $2 a$ (e.g., $n \times 0.808586 \mathrm{~Hz}, n=1,2,3,4)$. We assume that the pulsed fraction of the total detected events (cosmic-ray background + signal) scales approximately with the square root of the power ratio for a given harmonic (see Middleditch and Nelson 1976; also Gorham 1986).

From this assumption, we find that the pulsed fraction at the fundamental $(\sim 0.808 \mathrm{~Hz})$ varies by significant factor $(z 2.5 \sigma$, where $\sigma \approx 2 \%$ ) during the time series after eclipse. It is initially $\sim 8 \%$ in the first $\sim 20$ minutes, then drops to $\sim 3 \%$ during the middle third of the observation, and finally returns to $\sim 9 \%$ for the final $\sim 30$ minutes. In addition, we find that the pulsed fraction at the fourth harmonic $(\sim 3.23$ $\mathrm{Hz}$ ) is modulated at the $\sim 4 \sigma$ level, with a pulsed fraction of $\sim 0.4 \%$ early on becoming $\sim 9 \%$ within the final $\sim 30$ minutes of the time series. This harmonic modulation is evident in the two apparent components (broader sinusoid + sharp feature) of the light curve in Figure $2 c$. The remaining harmonics (second and third) were not present at significant levels.

Assuming a $5 \mathrm{kpc}$ distance to Her X-1, an emission solid angle of $\Omega \approx \pi \mathrm{sr}$, and a power-law emission spectrum with integral index -1.6 (similar to the cosmic-ray spectrum), the flux of $1.5 \times 10^{-10} \mathrm{~cm}^{-2} \mathrm{~s}^{-1}$ above $0.6 \mathrm{TeV}$ implies an integral $\gamma$-ray luminosity of $\sim 2.7 \times 10^{35} \mathrm{ergs} \mathrm{s}^{-1}$, which is comparable to the residual $\mathrm{X}$-ray luminosity as observed during X-ray eclipse. We note that the solid angle assumption above is based on the width of the broad feature in the light curve; if we instead use the sharp feature as an indicator of the beam width ( $\Omega \approx \pi / 5 \mathrm{sr}$ ), the average $\gamma$-ray luminosity would be closer to $\sim 5 \times 10^{34}$ ergs $\mathrm{s}^{-1}$ during this emission episode.

\section{CONCLUSIONS}

In the $1.25 \mathrm{hr}$ duration of the detectable $\gamma$-ray emission the pulsar has moved $\sim 20 \%$ of its way across the diameter $\left(\sim 5 \times 10^{11} \mathrm{~cm}\right)$ of the intervening companion star. Thus we wish to determine if it is possible that $\mathrm{TeV} \gamma$-rays, whether primaries or secondaries of some hadronic or electromagnetic cascade process, could penetrate the material of the star along the direct trajectory from Her X-1 to Earth. If we assume $\mathrm{HZ} \mathrm{Her} \mathrm{fills} \mathrm{its} \mathrm{Roche} \mathrm{lobe} \mathrm{with} \mathrm{a} \mathrm{mass} \mathrm{of} 2.2 \mathrm{M}_{\odot}$, and use a scale height of $4 \times 10^{8} \mathrm{~cm}$ (Deeter, Boynton, and Pravdo 1981) which we match to exterior densities of $N_{\mathrm{H}} \approx 10^{8} \mathrm{~cm}^{-3}$ (similar to the solar corona), then we estimate that column densities through the companion star's limb exceed 100 radiation lengths $\left(\sim 6000 \mathrm{~g} \mathrm{~cm}^{-2}\right)$ for both $\mathrm{TeV} \gamma$-rays and $\sim 10$ $\mathrm{TeV}$ proton primaries (required to produce approximately one $\mathrm{TeV} \gamma$-ray secondaries) within $\sim 7$ minutes after the eclipse transition. This eliminates the line-of-sight trajectory for either of these primary particles during the latter portion of the observation, and thus indicates that the $\gamma$-ray production site and X-ray source (diameter $<2 \times 10^{9} \mathrm{~cm}$ ) are not coincident.

It is interesting to note that, although line-of-sight column densities through the limb soon equal and exceed acceptable values for conversion of a primary particle beam to secondary $\gamma$-rays, there are few other regions of the system with adequate column densities to act efficiently as a "beam dump" for such conversion. For example, the mass stream emanating from the inner Lagrange point of $\mathrm{HZ} \mathrm{Her}$ has an estimated density of $N_{\mathrm{H}} \approx 6 \times 10^{12} \mathrm{~cm}^{-3}$ (Lubow and Shu 1975), giving only $\sim 0.3 \mathrm{~g} \mathrm{~cm}^{-2}$ per light-second of path length through it; also, the matter causing absorption dips in X-rays has a column density of typically $\sim 0.5 \mathrm{~g} \mathrm{~cm}^{-2}$ (Voges et al. 1985).

Eichler and Vestrand (1985) have suggested that such a beam dump process could account for the $>500 \mathrm{TeV}$ emission detected from Her X-1 by the Fly's Eye during a $\sim 40$ minute episode in 1983 (Baltrusaitis et al. 1985). If such a process is to apply to the emission reported in this Letter, it appears that the required densities $\left(\sim 10-100 \mathrm{~g} \mathrm{~cm}^{-2}\right.$ for efficient secondary $\gamma$-ray production from proton primaries; Stenger 1984) are possibly only found consistently in regions of the accretion disk or along the companion star limb. However, regardless of the production mechanism, the question of how coherent pulsations of $\mathrm{TeV}$ particles can survive so far into the $\mathrm{X}$-ray eclipse remains problematical.

We wish to thank David Lewis and John Learned for their comment and suggestion on this work, which was supported in part by the US Department of Energy.

\section{REFERENCES}

Baltrusaitas, R. M., et al. 1985, Ap. J. (Letters), 293, L69.

Davidsen, A., Henry, J. P., Middleditch, J., and Smith, H. E. 1972, Ap. J. ( Letters), 177, L97.

Deeter, J. E., Boynton, P. E., and Pravdo, S. H. 1983, Ap. J., 247, 1003.

Dowthwaite, J. C., Harrison, A. B., Kirkman, I. W., Macrae, H. J.,

Orford, K. J., Turver, K. E., and Walmsley, M. 1984, Nature 309, 691.

Eichler, D., and Vestrand, W. T. 1985, Nature, 318, 345.

Gorham, P. W. 1986, Ph.D. thesis, University of Hawaii.

Gorham, P. W., et al. 1986 Ap. J., in press (Paper I).

Groth, E. J. 1974, Ap. J., 192, 517.

Hillas, A. M. 1985, in Proc. 19th Cosmic Ray Conf. (La Jolla) (NASA

CP-2376), 3, 445 .
Leahy, D. A., Darbro, W., Elsner, R. F., Weisskopf, M. C., Sutherland, P. G., Kahn, S., and Grindlay, J. E. 1983, Ap. J., 266, 160. Lubow, S. H., and Shu, F. H. 1975, Ap. J., 198, 383.

Middleditch, J. 1976, Ph.D. thesis, University of California, Berkeley.

Middleditch, J., and Nelson, J. E. 1976, Ap. J., 208, 567.

Middleditch, J., Pennypacker, C. R., and Burns, M. S. 1983, Ap. J., 274, 313

Middleditch, J., Peutter, R. C., and Pennypacker, C. R. 1985, Ap. J., 292, 267.

Ögelman, H., Kahabka, P., Pietsch, W., Trümper, J., and Voges, W. 1985, Space Sci. Rev., 40, 347. 
Michael F. Cawley, Richard C. Lamb, and David F. Liebing: Department of Physics, Iowa State University, Ames, IA 50011 David J. Fegan and Niel A. Porter: Physics Department, University College, Stillorgan Road, Dublin 4, Ireland

KeN G. GibBS and Trevor C. WeEkes: Harvard-Smithsonian Center for Astrophysics, Whipple Observatory, P.O. Box 97,
Amado, AZ 85645-0097 Peter W. Gorham and Victor J. Stenger: Department of Physics and Astronomy, University of Hawaii, 2505 Correa Road,
Honolulu, HI 96822 